\title{
Perlecan-targeted nanoparticles for drug delivery to triple-negative breast cancer
}

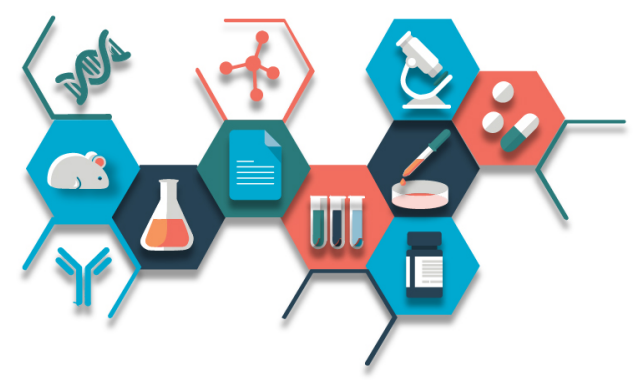

\author{
Vidhi Khanna ${ }^{1}$, Stephen Kalscheuer ${ }^{1}$, Ameya Kirtane', Wenqiu Zhang ${ }^{1}$ \& Jayanth \\ Panyam ${ }^{*, 1,2}$ \\ ${ }^{1}$ Department of Pharmaceutics, University of Minnesota, Minneapolis, MN 55455, USA \\ ${ }^{2}$ Masonic Cancer Center, University of Minnesota, Minneapolis, MN 55455, USA \\ *Author for correspondence: Tel.: +1 612624 0951; Fax: +1 612626 2125; jpanyam@umn.edu
}

\begin{abstract}
Aim: We previously developed two antibodies that bind to a cell surface protein, perlecan, overexpressed in triple-negative breast cancer (TNBC). The goal of this study was to investigate these antibodies as targeting ligands for nanoparticle-mediated drug delivery. Methods: Paclitaxel-loaded poly(D,L-lactide-coglycolide) nanoparticles were functionalized with antibodies using thiol-maleimide chemistry. Effect of antibody functionalization on therapeutic efficacy of drug-loaded nanoparticles was investigated using in vitro and in vivo models of TNBC. Results: The antibodies were covalently conjugated to nanoparticles without affecting antibody binding affinity or nanoparticle properties. Perlecan-targeted nanoparticles showed improved cell uptake, retention, cytotoxicity in vitro and enhanced tumor growth inhibition in vivo. Conclusion: The data presented here indicates that perlecan-targeted nanoparticles can improve tumor drug delivery to TNBC.
\end{abstract}

First draft submitted: 18 January 2019; Accepted for publication: 7 May 2019; Published online: 1 July 2019

Keywords: antibody $\bullet$ perlecan $\bullet$ polymeric nanoparticles $\bullet$ targeted drug delivery $\bullet$ triple-negative breast cancer

Breast cancer is the most common cause of malignancy in females, representing an astounding $30 \%$ of all diagnosed cases of cancer in the USA [1]. The diversity in subtypes of breast cancer, that are distinct in their response to various treatments, has made it difficult to develop a universally effective treatment for all patients. However, the availability of molecular markers for at least some of the subtypes has been a powerful tool for the development of subtype-specific therapies such as antihormonal therapies and HER2-targeted therapies. Unfortunately, treatment options for patients diagnosed with the triple-negative breast cancer (TNBC) subtype are limited to traditional chemotherapy, surgery and radiation [2]. However, traditional cytotoxic therapies are not tumor selective and are not effective, especially in patients with metastatic disease. Thus, there is a need for more effective and targeted therapies to treat TNBC.

We recently identified the overexpression of a cell surface protein, perlecan (HSPG2), in human TNBC [3]. HSPG2 is a large, $400 \mathrm{kDa}$ basement membrane protein that is heavily glycosylated and plays a role in tethering growth factors [4]. HSPG2 is critical for normal development [5,6]. Growth factors such as VEGF-A and FGF-2 are known to bind to HSPG2 (in addition to their own receptor) [7]. Thus, HSPG2 acts as a 'co-receptor'. Knockdown of perlecan in a metastatic prostate cancer cell line led to a reduction in growth factor-mediated cell proliferation [7]. A similar study in colon cancer demonstrated a reduction in tumor growth in vivo when perlecan was knocked down [8]. Our mRNA-based analysis showed that TNBC patients with high HSPG2 expression have a significantly lower survival rate compared with those with low HPSG2 expression, pointing to clinical significance of this novel target (Manuscript Submitted: S Kalscheuer, V Khanna, H Kim, S Li, D Sachdev, D Yang, A DeCarlo, J Panyam. Discovery of perlecan [HSPG2] as a therapeutic target in triple negative breast cancer).

The goal of the current studies was to investigate perlecan as a target for tumor-directed drug delivery. We previously developed two monoclonal antibodies (Clone 6 and AM6) that bind to perlecan expressing tumor cells with high affinity. Clone 6 has a $\mathrm{K}_{\mathrm{D}}$ value (equilibrium dissociation constant) of approximately $120 \mathrm{nM}$, whereas $\mathrm{AM} 6$ has a $\mathrm{K}_{\mathrm{D}}$ value of approximately $10 \mathrm{nM}$ [3]. In the current study, we fabricated poly(D,L-lactideco-glycolide) (PLGA) nanoparticles (NPs) encapsulating paclitaxel as a model chemotherapeutic agent and surface 
functionalized them with Clone 6 or AM6 (termed respectively as Clone 6 NPs or AM6 NPs). The therapeutic efficacy of perlecan-targeted NPs was investigated using in vitro and in vivo models of TNBC.

\section{Methods}

\section{Materials}

PLGA (50:50, 0.55-0.75 dL/g) was purchased from LACTEL Absorbable Polymers (AL, USA). Polylactidepolyethylene glycol with terminal maleimide functionalization (PLA-PEG-maleimide) (AI119) and PLGARhodamine (AV011) were purchased from PolySciTech (IN, USA). Polyvinyl alcohol (PVA, 30,000-70,000 MW), coumarin-6, sucrose, 2-imminothiolane hydrochloride and ethylenediaminetetraacetic acid (EDTA) were purchased from Sigma (MO, USA). Paclitaxel was purchased from Phytogen Life Science (British Columbia, Canada). Borate buffer stock was purchased from Alfa Aeser (MA, USA). Control Isotype IgG (Cat. No. 401114) was purchased from Calbiochem (MA, USA). The materials for SDS/PAGE were obtained from Bio-Rad (CA, USA). HPLC grade organic solvents were obtained from Fisher Scientific (PA, USA). All cell culture media and buffers (including phosphate buffered saline [PBS]) were purchased from Corning (MA, USA) or Life Technologies (CA, USA), unless otherwise specified. DI water was available through university resources.

\section{Preparation of PEG-maleimide-functionalized PLGA-NPs}

PLGA-NPs surface functionalized with maleimide-terminated PEG and loaded with paclitaxel and coumarin-6 or rhodamine were synthesized as described in an earlier publication [9]. Briefly, PLGA (30 mg) and coumarin-6 $(100 \mu \mathrm{g})$ or PLGA $(25 \mathrm{mg})$ and PLGA-rhodamine $(5 \mathrm{mg})$ along with paclitaxel $(5 \mathrm{mg})$ were dissolved in $1 \mathrm{ml}$ of chloroform. An oil-in-water emulsion was formed by emulsifying the polymer-drug solution in $8 \mathrm{ml}$ of $2.5 \% \mathrm{w} / \mathrm{v}$ aqueous PVA solution by probe sonication (18-24 W; Sonicator XL, Misonix, NY, USA) for 5 min over an ice bath. The diblock copolymer PLA-PEG-maleimide was dissolved in chloroform $(200 \mu \mathrm{l})$ and added dropwise to the above emulsion with stirring. The emulsion was stirred for 16-18 h under ambient conditions followed by $1 \mathrm{~h}$ under vacuum to remove the residual chloroform. NPs were washed twice by ultracentrifugation $(35,000 \mathrm{rpm}$ for 35 min at $4^{\circ} \mathrm{C}$, Optima XPN-80, Beckman, CA, USA) and reconstitution in DI water. The final NP dispersion was then stored at $4^{\circ} \mathrm{C}$ until conjugation reaction on the same day.

\section{Preparation \& purification of antibodies}

Expi293F Expression System by Life Technologies was used for the expression of human immunoglobulin (IgG) antibodies. Affinity purification of antibodies was carried out using Protein A Plus (Pierce Biotechnology, IL, USA) followed by buffer exchange into tris-buffered saline containing $5 \mathrm{mM}$ EDTA. Zeba ${ }^{\text {TM }}$ Spin Desalting Columns (87769, Pierce Biotechnology) were used for the buffer exchange step. Antibody stocks were stored at $-20^{\circ} \mathrm{C}$ in single-use aliquots until use. Once thawed, the samples were placed at $4{ }^{\circ} \mathrm{C}$ for short-term storage. Quality control evaluation involved resolution via SDS/PAGE for reduced and nonreduced samples and flow cytometry for confirmation of binding affinity. For flow cytometry, the antibodies were incubated with MDA-MB-231-LM2 cells at a concentration of $100 \mathrm{nM}$ for $1 \mathrm{~h}$ at $4^{\circ} \mathrm{C}$ on a rotating platform (Barnstead International, IA, USA), followed by three washes using FACS Buffer. Alexa 647 goat anti-human secondary antibody (Life Technologies) was added and the cells were incubated at $4^{\circ} \mathrm{C}$ for $30 \mathrm{~min}$ on a rotating platform, followed by three washes. The cells were re-suspended in flow buffer and placed on ice until analysis by flow cytometry (BD LSRFortessa, BD Biosciences, CA, USA).

\section{Optimization of antibody thiolation}

Purified antibody ( $300 \mu \mathrm{g}$ Clone 6, AM6 or isotype $\mathrm{IgG}$ control) was thiolated in $0.1 \mathrm{M}$ borate buffer $(\mathrm{pH}$ 8) with $5 \mathrm{mM}$ EDTA. The thiolation reaction was first optimized to ensure the formation of a thiol-maleimide bond as well as to minimize changes to the binding affinity of the antibodies. A 20-200-fold molar excess of the thiolating reagent 2-imminothiolane (also known as Traut's reagent) was used for the reaction. The samples were placed on a rotating platform for $2 \mathrm{~h}$ at room temperature, followed by desalting (Zeba Spin Desalting Columns 87769, Pierce Biotechnology) into PBS with $5 \mathrm{mM}$ EDTA. The number of thiols introduced per antibody molecule was determined using Amplite ${ }^{T M}$ fluorimetric thiol assay kit by AAT Bioquest (CA, USA). 


\section{Conjugation of antibody to NPs}

Once the thiolated antibodies were desalted, the samples were immediately added to the NP dispersion and placed on a rotating platform at $4^{\circ} \mathrm{C}$ to allow the conjugation reaction to take place overnight. NPs were then washed once by ultracentrifugation $\left(35,000 \mathrm{rpm}\right.$ for $35 \mathrm{~min}$ at $\left.4^{\circ} \mathrm{C}\right)$ and dispersed in $5 \mathrm{ml}$ DI water containing $30 \mathrm{mg}$ sucrose (lyoprotectant). The final dispersion was probe sonicated (30 s, 6-9 W) on an ice bath and centrifuged at $1000 \mathrm{rpm}$ for $5 \mathrm{~min}$ to pellet any large aggregates. The supernatant was then lyophilized (Labconco, FreeZone 4.5, MO, USA). The lyophilized product was stored in a desiccator at $4^{\circ} \mathrm{C}$ until use. An isotype human IgG was used to make control NPs, termed as isotype IgG NPs.

\section{Physiochemical characterization of NPs}

Hydrodynamic diameter and $\zeta$-potential of NPs in DI water were determined using DelsaTM Nano C (Beckman Coulter, Inc., CA, USA). Amplite Colorimetric Maleimide Quantitation kit by AAT Bioquest was used for maleimide quantification. To determine paclitaxel loading, NPs were dispersed in methanol $(1 \mathrm{mg} / \mathrm{ml})$ and incubated overnight at room temperature. The dispersion was then centrifuged at 13,000 rpm for $10 \mathrm{~min}$ and the supernatant was processed for HPLC analysis. Coumarin- 6 and/or paclitaxel loading was determined using an HPLC method previously described [10].

\section{Confirmation of antibody conjugation \& loading}

One milligram of NPs (Clone 6 conjugated, AM6 conjugated, isotype IgG conjugated or blank NPs) was dispersed in $40 \mu \mathrm{l}$ DI water and analyzed for protein concentration using bicinchoninic acid assay (Pierce BCA Protein Assay, IL, USA). Gel electrophoresis was used to confirm the formation of thiol-maleimide bond. $1 \mathrm{mg}$ of NPs was resuspended in $25 \mu \mathrm{LI}$ water. To each tube, $25 \mu \mathrm{l}$ of 95:5 2X gel loading buffer:2-mercaptoethanol (reduced samples) or $25 \mu \mathrm{l}$ of $2 \mathrm{X}$ gel loading buffer (nonreduced samples) was added. The samples were placed in a water bath at $90^{\circ} \mathrm{C}$ for $10 \mathrm{~min}$, centrifuged at $13,000 \mathrm{rpm}$ for $10 \mathrm{~min}$ and the supernatants were resolved on a $4-10 \%$ SDS/PAGE Criterion precast gel (Bio-Rad) along with the respective antibody controls.

\section{Cell culture}

Luciferase-expressing MDA-MB-231-LM2 (also referred to as LM2) cells were a gift from Dr Deepali Sachdev, University of Minnesota (USA). Cells between passages 2 and 10 were used for all the studies. Cells were cultured in minimum essential medium with $10 \% \mathrm{v} / \mathrm{v}$ fetal bovine serum and $1 \% \mathrm{v} / \mathrm{v}$ penicillin/streptomycin in a humidified atmosphere with $5 \% \mathrm{CO}_{2}$.

\section{Cell uptake of antibody-conjugated NPs analyzed by HPLC}

Coumarin-6-loaded NPs were used for this study. LM2 cells were seeded at 50,000 cells per well in a 24-well plate and allowed to adhere overnight. Cells were washed once with PBS and fresh media was added. The cells were then incubated at $4^{\circ} \mathrm{C}$ for $1 \mathrm{~h}$ with NPs at a final concentration of $100 \mu \mathrm{g} / \mathrm{ml}$ to allow binding to the target $(\mathrm{n}=3$ or 4$)$. The NP dispersion was then replaced with fresh media and the cells were placed at $37^{\circ} \mathrm{C}$ to allow internalization. The cells were lyzed at 60 min post addition of fresh media using $100 \mu$ radioimmunoprecipitation (RIPA) buffer. A part of the cell lysate $(30 \mu \mathrm{l})$ was used to determine protein concentration by bicinchoninic acid (BCA) assay and the rest was processed for HPLC analysis. Coumarin- 6 was extracted with $0.5 \mathrm{ml}$ methanol overnight. The samples were then centrifuged at $13,000 \mathrm{rpm}$ for $10 \mathrm{~min}$, and the supernatant was analyzed for coumarin- 6 concentration using HPLC [10].

\section{Cell uptake of antibody-conjugated NPs analyzed by flow cytometry}

Rhodamine-labeled NPs were used for this study. LM2 cells suspended in FACS buffer $(200,000 / 150 \mu \mathrm{l})$ were incubated with $50 \mu \mathrm{g}$ suspension of NPs on a rotating platform at $37^{\circ} \mathrm{C}$ for $1 \mathrm{~h}(\mathrm{n}=2)$. At the end of $1 \mathrm{~h}$, the cells were washed twice using FACS buffer, fixed using $4 \% \mathrm{v} / \mathrm{v}$ formaldehyde in FACS buffer, washed once, and then analyzed using flow cytometry (BD LSRFortessa H0081). In order to normalize data to rhodamine loading, fluorescence of the initial NP suspensions was measured using the IVIS Spectrum in vivo Imaging System (Caliper Lifesciences, MA, USA) or Spectramax i3x (Molecular Devices, CA, USA) fluorescence plate reader. 


\section{Exocytosis of antibody-conjugated NPs}

LM2 cells were seeded at 50,000 cells per well in a 24-well plate and allowed to adhere overnight. Cells were washed once with PBS and fresh media was added. The cells were then incubated at $37^{\circ} \mathrm{C}$ for $2 \mathrm{~h}$ with rhodamine-labeled NPs at a final concentration of $100 \mu \mathrm{g} / \mathrm{ml}$. After $2 \mathrm{~h}$, the cells were washed once and fresh media was added to allow exocytosis. The cells were lyzed at $0,15,30$ and $60 \mathrm{~min}$ post addition of fresh media, using $200 \mu \mathrm{l}$ RIPA buffer. Part of the cell lysate $(50 \mu \mathrm{l})$ was used to determine protein concentration by BCA assay and the rest was used to determine rhodamine fluorescence using Spectramax i3x (Molecular Devices) fluorescence plate reader.

\section{Competition experiment}

LM2 cells $(200,000)$ in suspension, were incubated at $4^{\circ} \mathrm{C}$ with $1 \mu \mathrm{M}$ (final concentration) of antibodies (or no antibody in case of control) for $1 \mathrm{~h}$ on a rotating platform. Cells were then washed three-times with FACS buffer and re-suspended in fresh FACS buffer. Antibody-conjugated NPs (rhodamine labeled; $50 \mu \mathrm{g}$ ) were added to the respective antibody treated/control tubes. The cells were once again incubated at $4{ }^{\circ} \mathrm{C}$ for $1 \mathrm{~h}$ on a rotating platform. Finally, the cells were washed three-times using FACS buffer, fixed using $4 \% \mathrm{v} / \mathrm{v}$ formaldehyde in FACS buffer and washed once. Flow cytometry analysis was carried out using BD LSRFortessa H0081.

\section{Cytotoxicity study}

LM2 cells were seeded at 2500 cells per well in a 96-well plate and allowed to adhere overnight. Treatments (free paclitaxel or paclitaxel-loaded NPs) were added the next day at 10, 30 and $50 \mathrm{nM}$ equivalent paclitaxel concentrations. The treatments were incubated for $6 \mathrm{~h}$, followed by removal of the treatments and addition of fresh media. Cell survival was analyzed at $72 \mathrm{~h}$ using CellTiter $96 \mathrm{AQ}_{\text {ueous }}$ One Solution Cell Proliferation Assay (Promega, WI, USA).

\section{In vivo efficacy study}

All experimental protocols using animals were reviewed and approved by University of Minnesota Institutional Animal Care and Use Committee (IACUC), and experiments were performed accordingly. Animals were housed in the research animal resources facility at the University of Minnesota under a $12 \mathrm{~h}$ light/dark cycle, under a temperature-controlled environment and were given access to food and water ad libitum. LM2 tumors were grafted in Balb/c homozygous nude mice (Jackson Labs, ME, USA). Once tumor volumes reached $100 \mathrm{~mm}^{3}$, six doses $(20 \mathrm{mg} / \mathrm{kg}$ equivalent dose of paclitaxel) of NPs were administered through tail vein injection once every $96 \mathrm{~h}$ ( $n=6-7$ per treatment group). One set of animals did not receive any treatment and served as untreated controls. Tumor volumes were measured every third day with an electronic caliper. Tumor volumes were calculated from the ellipsoid sphere equation $\mathrm{V}=\left(\mathrm{L}^{2} * \mathrm{~W}\right) / 2$, $\mathrm{L}$ being the longer measurement. Mice were sacrificed 30 days after the treatments begun. Two-way analysis of variance (ANOVA), with multiple comparison post-tests, was used to determine the statistical significance of the data.

\section{Results}

Optimization of antibody thiolation

Thiol-maleimide chemistry has been commonly used for hetero-functional crosslinking of proteins [11] as well as for the development of antibody-drug conjugates [12]. Here we used thiol-maleimide chemistry to conjugate antibodies to NPs (Figure 1).

We used '2-iminothiolane (Traut's reagent) to introduce reactive thiol groups on the lysine amino acids available in the $\operatorname{IgG}$ structure. A number of groups have used this strategy in the past [13,14]; however, the ratio of the thiolating reagent to antibody is critical and must be optimized for each antibody. Different molar ratios of the thiolating reagent to the antibody (20X, 50X, 100X and 200X) were tested. The number of thiols introduced per antibody molecule at each ratio is shown in Figure 2A. In order to confirm that thiolation of the antibody did not affect antibody binding, we carried out flow cytometry-based evaluation of the thiolated antibody. As depicted in Figure $2 \mathrm{~B}$, there was no change in the binding affinity upon thiolation, even at the highest concentration of the thiolating reagent. Based on these results, we used 200 -fold excess of the Traut's reagent to thiolate antibodies for conjugation with NPs. 


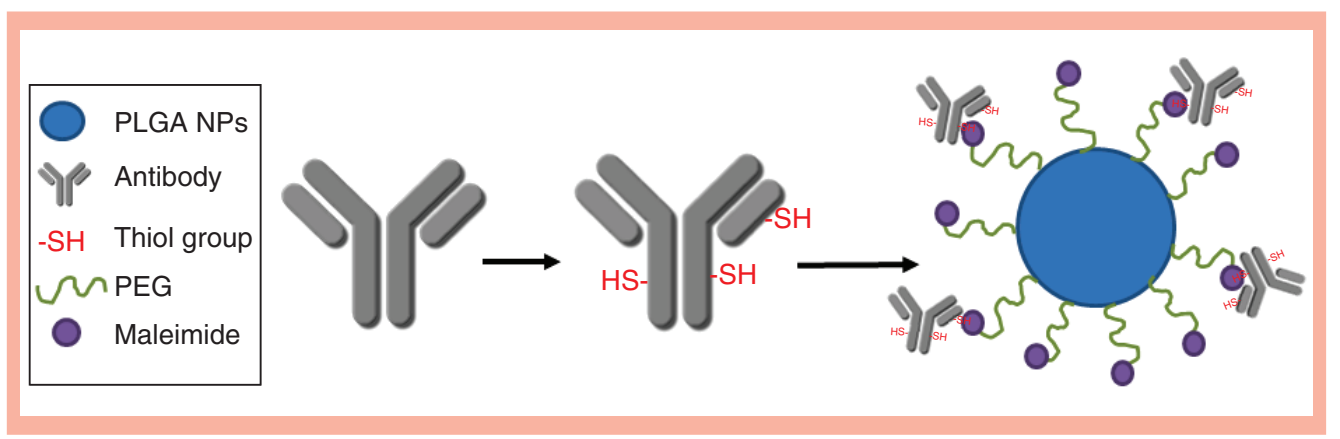

Figure 1. Schematic of antibody-conjugated nanoparticles preparation. Step 1: thiolation of antibody using 2-imminothiolane. Step 2: reaction of thiolated antibody with maleimide-functionalized nanoparticles. NP: Nanoparticle; PEG: Polyethylene glycol; PLGA: Poly(D,L-lactide-co-glycolide).

\begin{tabular}{|c|c|c|}
\hline Nanoparticles & Particle size (nm) (PDI) & $\zeta$-potential (mV) \\
\hline Blank NPs & $212(0.183)$ & -16.34 \\
\hline Clone 6 NPs & $209(0.213)$ & -13.22 \\
\hline AM6 NPs & $210(0.186)$ & -12.21 \\
\hline
\end{tabular}

\begin{tabular}{|l|l|}
\hline Table 2. Physicochemical characterization of nanoparticles $(\mathrm{n}=3-4$ batches). \\
\hline Maleimide content & Nanoparticle characterization \\
\hline Antibody loading & $0.11 \pm 0.036 \mu \mathrm{g} / \mathrm{mg}$ nanoparticles \\
\hline Conjugation efficiency & $8.95 \pm 3.55 \mu \mathrm{g} / \mathrm{mg}$ nanoparticles \\
\hline Paclitaxel loading & $70-100 \%$ \\
\hline
\end{tabular}

\section{Physiochemical characterization of NPs}

Average hydrodynamic size of NPs was determined to be $209 \pm 2.5 \mathrm{~nm}$. $\zeta$-potential was $-13.4 \pm 2.05 \mathrm{mV}$ (Table 1). Presence of antibody did not significantly influence the particle size or $\zeta$-potential. We used interfacial activity-assisted surface functionalization [15] to introduce maleimide groups on the surface of NPs. The maleimide content on NPs was confirmed prior to conjugation of the antibody and was found to be $0.11 \pm 0.04 \mu \mathrm{g} / \mathrm{mg} \mathrm{NP}$. Paclitaxel encapsulation efficiency was $>90 \%$ and average loading was $119 \pm 13 \mu \mathrm{g} / \mathrm{mg}$ of NPs.

\section{Confirmation of antibody conjugation \& loading}

Antibody content on NPs was determined using the BCA assay to be $9 \pm 4 \mu \mathrm{g}$ protein/mg NPs (Table 2). Based on this, it is estimated that there are approximately 40 antibody molecules per particle. In order to confirm that the antibody is covalently conjugated to NPs, we used SDS/PAGE. The thiol bonds between the heavy and light chains of the antibody were reduced using 2-mercaptoethanol. The thiol-maleimide undergoes retro and exchange reactions in the presence of thiols; however, these reactions have half lives ranging between 20 and $80 \mathrm{~h} \mathrm{[16].}$

Thus, when antibody-conjugated NPs were subjected to the reduction step, only half the antibody molecule was released from the surface of NPs (the light chain in this case, corresponding to $25 \mathrm{kDa}$ ), as observed in lanes 5,7 and 9 in Figure 2C. Without the reduction step, we observed no bands (lanes 4,6 and 8). From these results, we concluded that the antibodies were covalently conjugated to NPs, primarily via the heavy chains.

\section{Cell uptake of antibody-conjugated NPs}

Uptake of antibody-conjugated NPs was studied in LM2 cells, which have been previously established in our laboratory as a perlecan-positive cell line [3]. We initially determined the effect of antibody conjugation on paclitaxel delivery to the cells. We observed a 1.3-fold improvement in paclitaxel delivery with Clone 6 NPs (nonsignificant) 


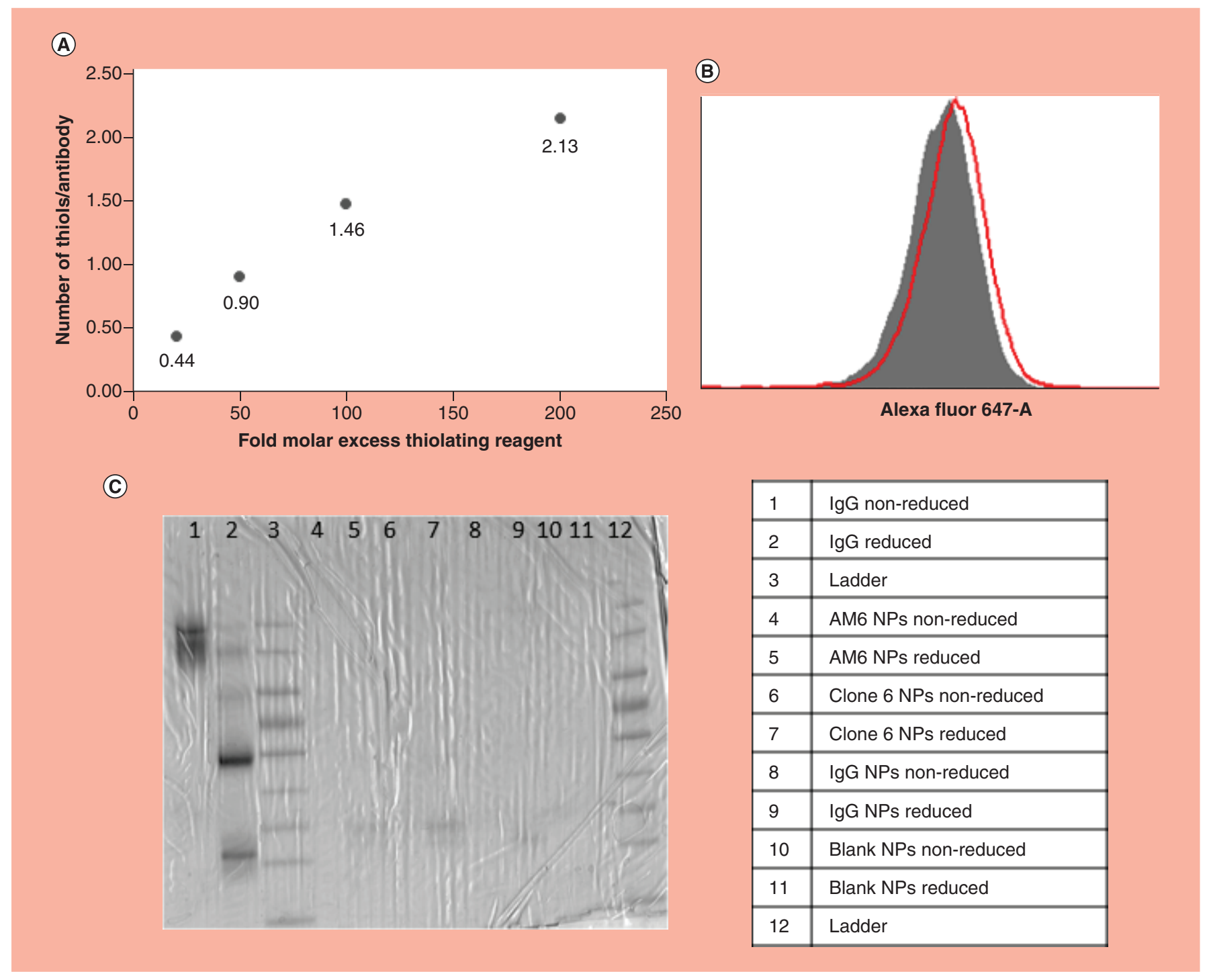

Figure 2. Optimization of antibody thiolation and conjugation to nanoparticles. (A) Number of thiols/antibody versus concentration of thiolating reagent. Number of thiols/antibody increases with increasing concentration of thiolating reagent; (B) AM6 unmodified binding to LM2 cells (gray), AM6 thiolated at $200 \times$ binding to LM2 cells. The thiolation reaction at $20 \times$ did not cause changes in antibody binding to the target cells; (C) SDS/PAGE evaluation of antibody-conjugated nanoparticles, through reduction and nonreduction, for confirmation of thiol-maleimide bond. For antibody-conjugated nanoparticles, only half of the antibodies are released when the nanoparticles were reduced (lanes 5, 7 and 9), whereas no antibody was released without reduction (lanes 4, 6 and 8).

NP: Nanoparticle.

and 1.7-fold improvement in the case of AM6 NPs $(\mathrm{p}<0.001)$ relative to that of IgG NPs (Figure 3A). Improved internalization of perlecan-targeted NPs was also verified by flow cytometry. For this purpose, we used PLGA polymer that was covalently conjugated to rhodamine. A twofold difference was observed between IgG and Clone 6 NPs (non-significant), whereas a five-fold difference was observed between isotype IgG NPs and AM6 NPs $(\mathrm{p}<0.001$; Figure 3B).

\section{Competition experiment}

In order to confirm that the improved uptake of antibody-conjugated NPs could be attributed to specific binding, we conducted a competition experiment. LM2 cells were pretreated with high concentrations of the respective antibodies at $4^{\circ} \mathrm{C}$ to saturate the target. Free Clone 6 and $\mathrm{AM} 6$ antibodies, but not the isotype control $\mathrm{IgG}$, reduced the cellular uptake of respective antibody-conjugated NPs (Figure 4A). The low reduction in uptake in this 
(A)

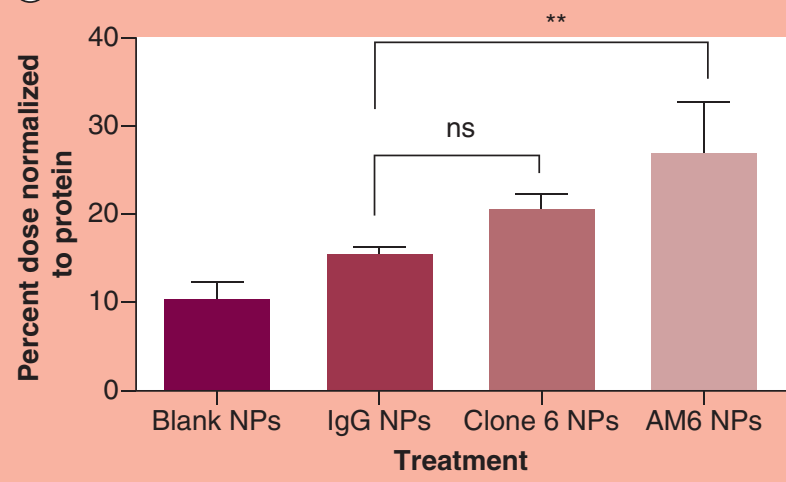

(B)

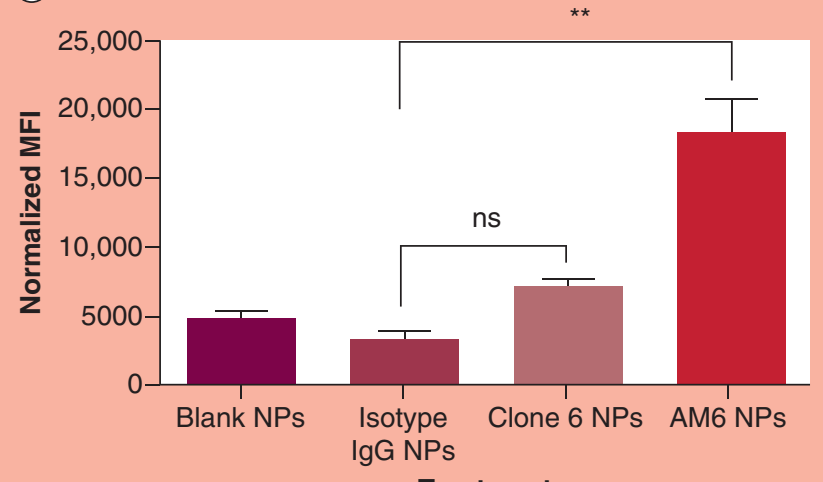

Treatment

Figure 3. Uptake of antibody-conjugated nanoparticles in LM2 cells. (A) Effect of various treatments on paclitaxel accumulation in the cells. Paclitaxel concentration was determined by HPLC and normalized to cell protein (HPLC). Statistical significance was calculated by one-way ANOVA with multiple comparisons. (B) cellular uptake of rhodamine-labeled nanoparticles measured by flow cytometry. $* * p<0.01$.

HPLC: High-performance liquid chromatography; MFI: Median fluorescence intensity; NP: Nanoparticle; ns: Nonsignificant.

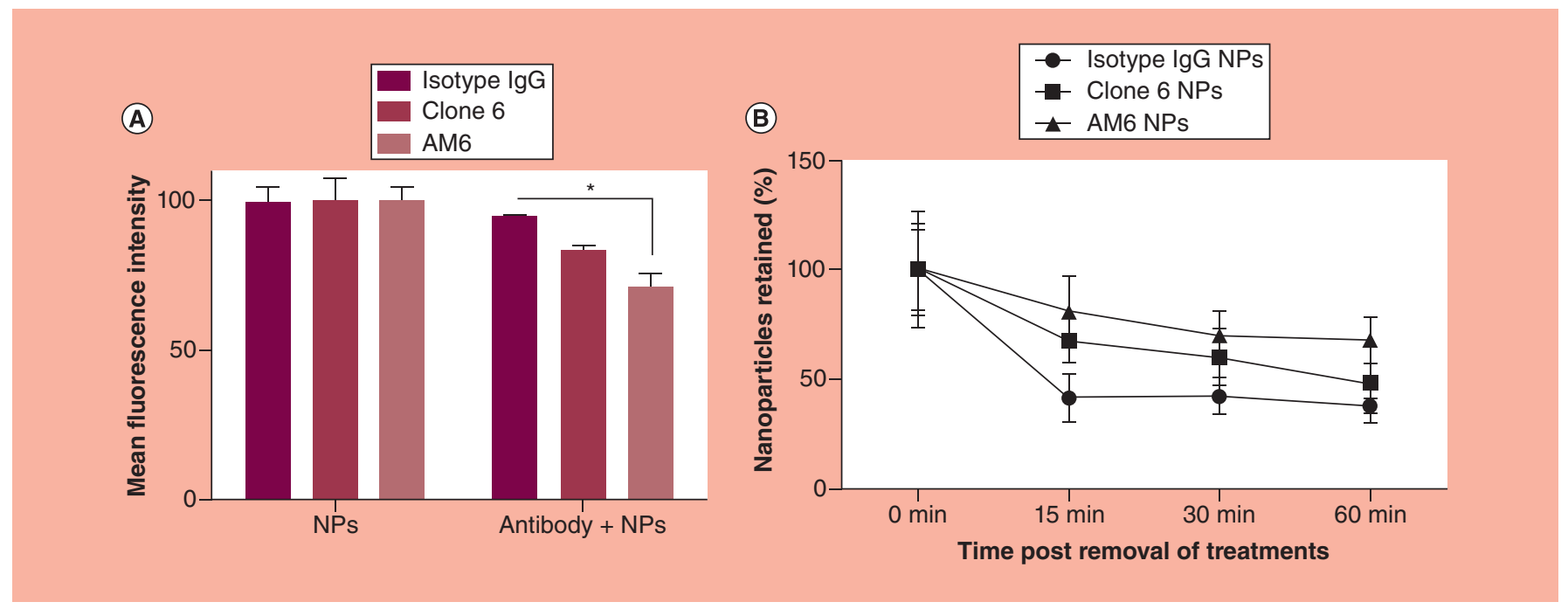

Figure 4. Specific uptake and retention of antibody-conjugated nanoparticles in LM2 cells. (A) Competition experiment with free antibody and antibody-conjugated nanoparticles to demonstrate specific uptake. Statistical significance was calculated based on a two-way ANOVA with multiple comparisons. * $p<0.05$. (B) Exocytosis of isotype IgG nanoparticles, Clone 6 nanoparticles and AM6 nanoparticles. Two-way ANOVA with multiple comparisons was significant for isotype IgG nanoparticles versus AM6 nanoparticles at 15 $\min (p<0.01), 30 \mathrm{~min}(p<0.05)$ and $60 \mathrm{~min}(p<0.05)$; isotype IgG versus Clone 6 nanoparticles was significant only at 15 min $(p<0.05)$. IgG: Immunoglobulin; NP: Nanoparticle.

competition experiment may be attributed to the fact that the antibody concentrations in the pretreatment step may not have been close to saturation. As a result, there may still have been perlecan epitopes available for binding with the targeted NPs. In addition, NPs are known to have non-specific uptake into cells, which likely accounts for a significant fraction of the NPs taken up [17].

\section{Exocytosis of antibody-conjugated NPs}

Presence of a targeting ligand on NPs is not only expected to improve internalization into cells but also the intracellular retention of NPs [18-20]. This can provide sustained concentrations of the drug within the cells, resulting in improved cytotoxic effect. We observed an increase in the fraction of NPs retained within the cells for AM6 NPs (Figure 4B) relative to isotype IgG NPs. The percentage nanoparticles in Figure 4B are normalized to 
(A)

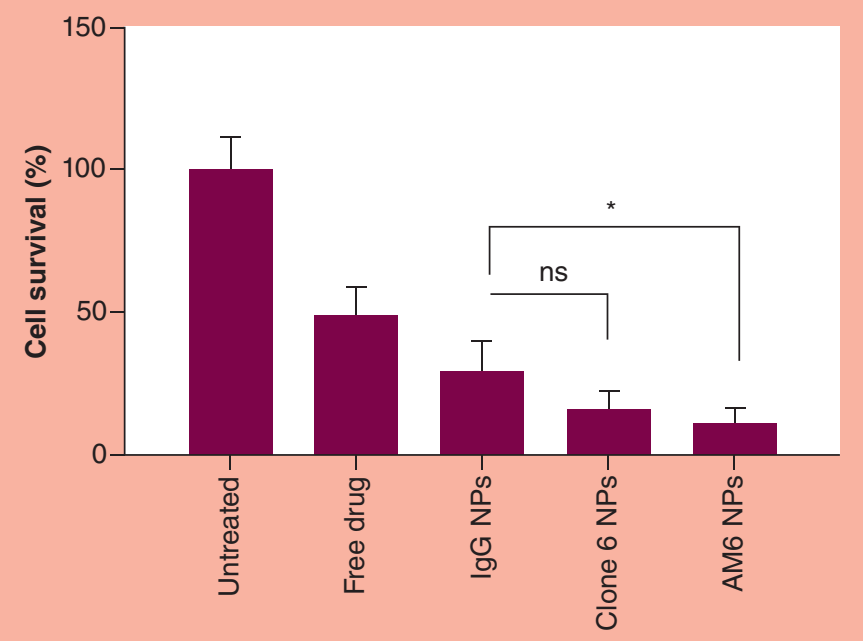

(B)

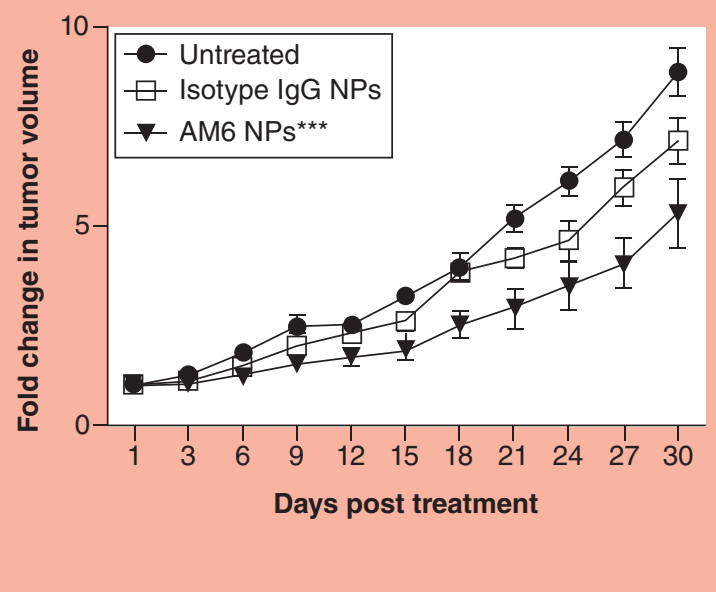

Figure 5. Improved drug delivery using antibody-conjugated nanoparticles. (A) In vitro cytotoxicity study in LM2 cells with paclitaxel-loaded nanoparticles. Statistical significance was calculated using a one-way ANOVA with multiple comparisons. ns (nonsignificant) $p>0.05,{ }^{*} p<0.05$. (B) In vivo tumor inhibition study in the MDA-MB-231-LM2 orthotopic tumor mouse model. Error bars shown are for standard error of the mean. Statistical significance is based on two-way ANOVA with multiple comparisons.

$* * * p<0.001$ based on comparison between isotype immunoglobulin nanoparticles and AM6 nanoparticles on day 30. NP: Nanoparticle; ns: Nonsignificant.

\section{Table 3. IC50 values for in vitro cytotoxicity study with paclitaxel-loaded nanoparticles.}

\begin{tabular}{|ll|}
\hline Treatment & $I_{50}(\mathrm{nM})$ \\
\hline Free drug & 11.73 \\
\hline Isotype IgG NPs & 5.32 \\
\hline Clone 6 NPs & 2.45 \\
\hline AM6 NPs & 1.49 \\
\hline IgG: Immunoglobulin; NP: Nanoparticle. \\
\hline
\end{tabular}

the amount of NPs present in the cells at the end of the (uptake) incubation period and shows that targeted NPs have improved retention within target cells.

\section{Cytotoxicity studies on MDA-MB-231-LM2}

In order to determine whether improved delivery of paclitaxel observed with perlecan-targeted NPs (Figure 3A) translates into improved efficacy, we conducted a cytotoxicity study in LM2 cells. Five treatment groups were considered - no treatment, free paclitaxel, paclitaxel-loaded isotype IgG NPs, paclitaxel-loaded Clone 6 NPs and paclitaxel-loaded AM6 NPs (Figure 5A). Maximum cell kill was observed in the case of Clone 6 NPs and AM6 NPs. AM6 NPs showed significantly higher cell kill as compared with isotype IgG NPs (p < 0.05). AM6 NPs had an $\mathrm{IC}_{50}$ approximately 3.5-fold lower than that of Isotype IgG NPs (Table 3). The superior activity of AM6 NPs when compared with Clone 6 NPs is likely attributed to the approximately tenfold difference in affinity between the two antibodies. We also observed an improvement in cell kill with paclitaxel in control NPs relative to free paclitaxel $(\mathrm{p}<0.05)$ as shown in previous studies [4].

\section{In vivo efficacy study}

In order to test the ability of AM6 NPs to improve drug delivery in vivo, we carried out an efficacy study with paclitaxel-loaded NPs in the MDA-MB-231-LM2 orthotopic mouse tumor model. NPs are known to have improved accumulation in the tumor due to the enhanced permeability and retention (EPR) effect [21]. Targeted NPs on the other hand have improved internalization into the tumor cells in addition to the EPR effect [18,19]. Based on improved cellular uptake, retention and cytotoxicity with AM6 NPs relative to Clone 6 NPs, we tested 
AM6 NPs in vivo. We observed improved tumor growth inhibition with AM6 NPs relative to isotype IgG NPs $(\mathrm{p}<0.001)$. On day 30, the untreated animals reached an average tumor volume of approximately $950 \mathrm{~mm}^{3}$. Isotype IgG NPs showed 18\% tumor inhibition. On the other hand, AM6 NPs showed 44\% tumor inhibition at the end of the study.

\section{Discussion}

Early diagnosis and the availability of targeted agents have resulted in improved outcomes for patients diagnosed with breast cancer. However, patients diagnosed with TNBC still have only a few treatment options available to them. Using phage display, our laboratory successfully developed two antibodies (Clone 6 and AM6) that bind to human triple negative breast cancer (TNBC cells) with varying affinities. The cognate ligand for these antibodies is perlecan, a member of a large family of proteoglycans known as heparan sulfate proteoglycans [22]. It is a critical proteoglycan, tethering a number of essential growth factors $[5,6,23,24]$. A previous publication from our lab showed that perlecan is overexpressed on metastatic tumor cells and that the AM6 antibody is able to accumulate selectively in the tumor [3].

We hypothesized that Clone 6 and AM6 antibodies could be used as ligands for targeted drug delivery to TNBC cells. Nontargeted NPs accumulate in tumor passively through the EPR effect [21]. Tumors have leaky vasculature compared with normal tissues, which allows for enhanced tumor accumulation of NPs. The poorly developed lymphatic drainage allows for extended retention of the delivery system within the tumor. Abraxane (albumin-bound paclitaxel) and Doxil (liposomal doxorubicin) are prototypical examples of nontargeted systems, which have been approved by the US FDA [25]. Both formulations have improved safety profiles compared with the respective free drugs. Incorporation of targeting ligands on the surface of nano drug-delivery systems allows for their specific binding to tumor cells and can result in significantly improved internalization as well as intratumoral retention of the delivery system [18-20].

In our studies, PLGA-NPs with maleimide-terminated PEG on the surface were fabricated using the interfacial activity-assisted surface functionalization technique [15]. The PEG layer served to prolong half-life in the circulation by forming a hydrophilic shield on the particles and preventing adsorption of opsonins and consequent phagocytosis by ubiquitous macrophages [26]. The maleimide group served as the reactive functional group on the NP surface. In order to conjugate the antibodies to the surface of NPs, we used thiol-maleimide chemistry [11]. Anhorn et al. [14] used thiolated trastuzumab for synthesis of antibody-conjugated NPs. Using a similar approach to the one we used here, they thiolated the antibody using 50-fold molar excess of 2 -imminothiolane and yet observed $40-50 \%$ of adsorptive binding (as opposed to covalent conjugation). Steinhauser $e t$ al. [13] obtained only 0.7 thiols per antibody using a similar protocol and also observed that some of the antibody was physically adsorbed onto NPs. Thus, in order to avoid adsorption issues and to improve antibody-mediated binding to target cells, we optimized the reaction conditions and were able to introduce between two and three thiols per antibody. Final characterization of the antibody-NP conjugates revealed a stable bond between antibody and NPs. The key physical characteristics of NPs were not significantly affected by presence of the antibody, likely due to the relatively small number of antibody molecules conjugated per NP.

We observed a 1.7-5-fold improvement in cell uptake with AM6 NPs. The variability in fold differences between the HPLC and flow cytometry experiments could be because of the following. In the case of HPLC studies, we measured total paclitaxel (free and NP encapsulated); contrary to drug loaded in NPs, free drug can easily efflux and/or diffuse out of the cells, resulting in reduced concentrations. Thus, paclitaxel concentrations may not directly reflect NP concentrations in the cells. For flow cytometry studies, we used rhodamine covalently conjugated to PLGA. In this case, we directly measure the fluorescence associated with NPs. Previous studies show that cellular uptake is affected by a number of factors including intrinsic antibody affinity, the density of antibody on the surface of NPs and the density of the target receptor on the cell surface [27]. Following internalization, NPs must be retained long enough so they are able to release the drug over time to achieve sustained intracellular drug concentrations. In addition, it has also been shown that non-targeted PLGA-NPs undergo exocytosis, resulting in the clearance of $>65 \%$ of the internalized dose within $30 \mathrm{~min}$ of removing the extracellular concentration gradient [28]. We observed that conjugation of the targeted antibody results in an increase in the intracellular retention of NPs; this is attributed to the ability of NPs to 'hold on' to the target inside the cell.

There was a significant improvement in the in vitro cytotoxicity of paclitaxel when delivered using targeted NPs that can likely be attributed to both increased cellular uptake and longer retention of targeted NPs, resulting in higher drug concentrations inside the cell. We also observed a difference between free drug and control NPs. This 
is likely due to nonspecific endocytosis of NPs, resulting in improved subcellular localization (closer to the target site of action inside the cell) [21,29]. NPs also enable sustained release of the drug, thus providing a consistent dose as opposed to the transient increase in drug concentration with free drug treatment. In agreement with the results of the in vitro studies, we observed significantly improved tumor growth inhibition in vivo with AM6 NPs relative to nontargeted isotype IgG NPs. Our observation of targeted NPs showing improved therapeutic efficacy is similar to previous reports $[9,30]$. However, it is important to note that perlecan has not been evaluated previously as a target for drug delivery.

In summary, the studies presented here show that perlecan-targeted NPs improve tumor-targeted drug delivery. Detailed pharmacokinetic and biodistribution studies are needed to further demonstrate improved tumor targeting of AM6 NPs. Optimization of NP characteristics (size, drug loading, PEG and antibody density) as well as improving antibody affinity could further enhance the therapeutic efficacy of perlecan-targeted NPs. Finally, LM2 cells express moderately high levels of perlecan. Additional studies utilizing cell lines with higher perlecan expression and those with minimal or no perlecan expression would be important to further establish the utility of this approach.

\section{Conclusion}

In conclusion, perlecan is a promising new target for the development of targeted therapies for the treatment of TNBC.

\section{Future perspective}

A quick search in the NIH database for clinical trials returns more than 180 hits for TNBC. This number provides an insight into the intensive interest in advancing new therapies for this disease. Lack of targetable markers, which can be leveraged for drug delivery, remains an important limitation. The data presented here suggest that perlecan could be a potential target for improving tumor drug delivery in TNBC.

\section{Summary points}

- Perlecan (HSPG2) is a basement membrane protein that is overexpressed in several different types of cancers, including triple-negative breast cancer.

- Clone 6 and AM6 are human, full-length immunoglobulins that bind specifically to perlecan and have been developed in our lab.

- We hypothesized that antiperlecan antibodies could be used to improve the tumor accumulation of nanoparticles.

- Clone 6 and AM6 were conjugated to nanoparticles using thiol-maleimide chemistry.

- Nanoparticles were characterized for their physicochemical properties including size, $\zeta$-potential, maleimide content, protein content and conjugation chemistry.

- Clone 6 and AM6-conjugated nanoparticles showed improved cellular uptake and retention as well as improved cell kill in in vitro assays.

- Based on promising in vitro data, we also carried out an efficacy study in an MDA-MB-231-LM2 orthotopic mouse model.

- AM6-conjugated nanoparticles showed significantly enhanced tumor growth inhibition as compared with isotype immunoglobulin nanoparticles.

Financial \& competing interests disclosure

J Panyam, V Khanna and S Kalscheuer have a US patent application disclosing the composition of the antibody reagents and their use described in this manuscript. Funding was provided by the Community of Pharmaceutical Development (to J Panyam) and NIH EB 019893 (to J Panyam). The authors have no other relevant affiliations or financial involvement with any organization or entity with a financial interest in or financial conflict with the subject matter or materials discussed in the manuscript apart from those disclosed.

No writing assistance was utilized in the production of this manuscript. 
Ethical conduct of research

The authors state that they have obtained appropriate institutional review board approval for all animal experimental investigations.

\section{References}

Papers of special note have been highlighted as: $\bullet$ of interest

1. American Cancer Society. Cancer facts \& figures 2017 (2017) www.cancer.org/research/cancer-facts-statistics/all-cancer-facts-figures/cancer-facts-figures-2017.html

2. Liedtke C, Mazouni C, Hess KR et al. Response to neoadjuvant therapy and long-term survival in patients with triple-negative breast cancer. J. Clin. Oncol. 26(8), 1275-1281 (2008).

3. Kalscheuer S. Humanized Antibody Development Using Phage Display: Applications to Solid Tumor Metastasis (PhD thesis). University of Minnesota, MN, USA (2016). https://conservancy.umn.edu/handle/11299/185603

- Describes the development of Clone 6 and AM6 antibodies and their initial characterization; both these antibodies have been used in the publication presented here.

4. Murdoch AD, Dodge GR, Cohen I, Tuans RS, Iozzo RV. Primary structure of the human heparan sulfate proteoglycan from basement membrane (HSPG2/Perlecan). J. Biol. Chem. 267(12), 8544-8557 (1992).

5. Olsen BR. Life without perlecan has its problems. J. Cell Biol. 147(5), 909-911 (1999).

6. Aviezer D, Hecht D, Safran M, Eisinger M, David G, Yayon A. Perlecan, basal lamina proteoglycan, promotes basic fibroblast growth factor-receptor binding, mitogenesis, and angiogenesis. Cell 79(6), 1005-1013 (1994).

7. Savorè C, Zhang C, Muir C et al. Perlecan knockdown in metastatic prostate cancer cells reduces heparin-binding growth factor responses in vitro and tumor growth in vivo. Clin. Exp. Metastasis 22(5), 377-390 (2005).

- Examines the implications of perlecan knockdown on the growth of a prostate cancer cell line. Perlecan knockdown reduced the sensitivity of the cell line to growth factor (FGF-2 and VEGF-A) and reduced their growth rate in vivo.

8. Sharma B, Handler M, Eichstetter I, Whitelock JM, Nugent MA, Iozzo RV. Antisense targeting of perlecan blocks tumor growth and angiogenesis in vivo. J. Clin. Invest. 102(8), 1599-1608 (1998).

9. Swaminathan SK, Roger E, Toti U, Niu L, Ohlfest JR, Panyam J. CD133-targeted paclitaxel delivery inhibits local tumor recurrence in a mouse model of breast cancer. J. Control. Rel. 171(3), 280-287 (2013).

- Describes the development of CD133-targeted nanoparticles for tumor drug delivery. The methods used for antibody conjugation are similar to those adapted here.

10. Patil Y, Swaminathan S, Sadhukha T, Ma L, Panyam J. The use of nanoparticle-mediated targeted gene silencing and drug delivery to overcome tumor drug resistance. Biomaterials 31(2), 612-626 (2011).

11. Hermanson GT. Antibody modification and conjugation. In: Bioconjugate Techniques (2nd Edition). Academic Press, Cambridge, USA, 783-823 (2008).

12. Jain N, Smith SW, Ghone S, Tomczuk B. Current ADC linker chemistry. Pharm. Res. 32(11), 3526-3540 (2015).

13. Steinhauser I, Spänkuch B, Strebhardt K, Langer K. Trastuzumab-modified nanoparticles: optimisation of preparation and uptake in cancer cells. Biomaterials 27(28), 4975-4983 (2006).

- Describes the development of antibody-conjugated nanoparticles targeting the HER2 antigen, as well as formulation optimization, characterization.

14. Anhorn MG, Wagner S, Kreuter J, Langer K, Von Briesen H. Specific targeting of HER2 overexpressing breast cancer cells with doxorubicin-loaded trastuzumab-modified human serum albumin nanoparticles. Bioconjug. Chem. 19(12), 2321-2331 (2008).

- Describes the development of antibody-conjugated serum albumin nanoparticles targeting the HER2 antigen, as well as formulation optimization, characterization.

15. Patil YB, Toti US, Khdair A, Ma L, Panyam J. Single-step surface functionalization of polymeric nanoparticles for targeted drug delivery. Biomaterials 30(5), 859-866 (2009).

16. Baldwin AD, Kiick KL. Tunable degradation of maleimide - adducts in reducing environments. Bioconjug. Chem. 22(10), 1946-1953 (2011).

17. Gao H, Yang Z, Zhang S et al. Ligand modified nanoparticles increases cell uptake, alters endocytosis and elevates glioma distribution and internalization. Sci. Rep. 3, 2534 (2013).

- Investigates the effects of ligand conjugation on the targeting mechanisms of nanoparticles using IL-13 peptide.

18. Bartlett DW, Su H, Hildebrandt IJ, Weber WA, Davis ME. Impact of tumor-specific targeting on the biodistribution and efficacy of siRNA nanoparticles measured by multimodality in vivo imaging Derek. Proc. Natl Acad. Sci. USA 104(39), 15549-15554 (2007).

19. Kirpotin DB, Drummond DC, Shao Y et al. Antibody targeting of long-circulating lipidic nanoparticles does not increase tumor localization but does increase internalization in animal models. Cancer Res. 66(13), 6732-6740 (2006).

20. Oyewumi MO, Yokel RA, Jay M, Coakley T, Mumper RJ. Comparison of cell uptake, biodistribution and tumor retention of folate-coated and PEG-coated gadolinium nanoparticles in tumor-bearing mice. J. Control. Rel. 95(3), 613-626 (2004). 
21. Murakami M, Cabral H, Matsumoto $\mathrm{Y}$ et al. Improving drug potency and efficacy by nanocarrier-mediated subcellular targeting. Sci. Transl. Med. 3(64), 1-11 (2011).

22. Iozzo RV. Matrix proteoglycans: from molecular design to cellular function. Annu. Rev. Biochem. 67(1), 609-652 (1998).

23. Zoeller JJ, Whitelock JM, Iozzo RV. Perlecan regulates developmental angiogenesis by modulating the VEGF-VEGFR2 axis. Matrix Biol. 28(5), 284-291 (2009).

24. Melrose J, Roughley P, Knox S, Smith S, Lord M, Whitelock J. The structure, location, and function of perlecan, a prominent pericellular proteoglycan of fetal, postnatal, and mature hyaline cartilages. J. Biol. Chem. 281(48), 36905-36914 (2006).

25. Kamaly N, Xiao Z, Valencia PM, Radovic-Moreno AF, Farokhzad OC. Targeted polymeric therapeutic nanoparticles: design, development and clinical translation. Chem. Soc. Rev. 41(7), 2971-3010 (2012).

26. Harris JM, Chess RB. Effect of pegylation on pharmaceuticals. Nat. Rev. Drug Discov. 2(3), 214-221 (2003)

27. Zhou Y, Drummond DC, Zou H et al. Impact of single-chain Fv antibody fragment affinity on nanoparticle targeting of epidermal growth factor receptor-expressing tumor cells. J. Mol. Biol. 371(4), 934-947 (2007).

28. Panyam J, Labhasetwar V. Dynamics of endocytosis and exocytosis of poly(D,L-lactide-co-glycolide) nanoparticles in vascular smooth muscle cells. Pharm. Res. 20(2), 212-220 (2003).

29. Omelyanenko V, Kopekova P, Gentry C, Kopecek J. Targetable HPMA copolymer-adriamycin conjugates. Recognition, internalization, and subcellular fate. J. Control. Rel. 53(1-3), 25-37 (1998).

30. Cheng J, Teply BA, Sherifi I et al. Formulation of functionalized PLGA-PEG nanoparticles for in vivo targeted drug delivery. Biomaterials 28(5), 869-876 (2007). 